\title{
Multiple Time Resolution Unit Commitment for Short-Term Operations Scheduling Under High Renewable Penetration
}

\author{
Emmanouil A. Bakirtzis, Student Member, IEEE, Pandelis N. Biskas, Member, IEEE, \\ Dimitris P. Labridis, Senior Member, IEEE, and Anastasios G. Bakirtzis, Senior Member, IEEE
}

\begin{abstract}
This paper introduces the idea of unified unit commitment and economic dispatch modeling within a unique tool that performs economic dispatch with up to 24 -hour look-ahead capability. The tool provides financially binding dispatch and ex-ante locational marginal prices (LMPs) for the next 5-min interval and advisory commitment, dispatch schedule and prices for the remaining scheduling horizon. Variable time resolution and variable modeling complexity are used in order to reduce computational requirements. A finer time resolution and detailed modeling are used during the first hours of the scheduling horizon while coarser time resolution and simplified modeling during the last ones. The viability of the method for medium-sized systems is demonstrated through its application to the Greek power system.
\end{abstract}

Index Terms-Economic dispatch, mixed integer linear programming, renewable generation, unit commitment.

\section{NOMENCLATURE}

\section{A. Indices and Sets}

$b \in \mathcal{B}^{c} \quad$ Overloaded branches during contingency $c$.

$c \in C^{q} \quad$ Branch contingencies in model of complexity $q$.

$f \in \mathcal{F}^{i} \quad$ Steps of the marginal cost function of unit $i$.

$i \in I \quad$ Generating units.

$i \in I^{\mathcal{T H}} \quad$ Thermal generating units $I^{\mathcal{T H}} \subseteq I$.

$i \in I^{\mathcal{H} \mathcal{Y}} \quad$ Hydroelectric generating units $I^{\mathcal{H} \mathcal{Y}} \subseteq I$.

$i \in I^{\mathcal{L S}} \quad$ Long-start generating units $I^{\mathcal{L S}} \subseteq I^{\mathcal{T} \mathcal{H}}$.

$j \in \mathcal{J} \quad$ Unit start-up types $\mathcal{J}=\{h, w, c\}(=$ hot, warm, cold).

$\ell \in \mathcal{L} \quad$ Loads.

$m \in \mathcal{M} \quad$ Reserves types $\mathcal{M}=$ $\{1+, 1-, 2+, 2-, 3 S, 3 N S\}$, where $m=1+$ : primary-up, $m=1-$ : primary-down, $m=2+$ : secondary-up, $m=2-$ : secondary-down, $m=3 \mathrm{~S}$ : tertiary spinning, $m=3 \mathrm{NS}$ : tertiary non-spinning.

Manuscript received November 07, 2012; revised March 05, 2013 and June 11, 2013; accepted August 07, 2013. Date of publication September 10, 2013; date of current version December 16, 2013. This work was supported by the Greek Secretariat of Research and Technology under Grant 1522-ARISTEIA. Paper TPWRS-01247-2012.

The authors are with the Department of Electrical and Computer Engineering, Aristotle University of Thessaloniki, 54124 Thessaloniki, Greece (e-mail: emmpakir@auth.gr; pbiskas@auth.gr; labridis@auth.gr; bakiana@eng.auth.gr)

Color versions of one or more of the figures in this paper are available online at http://ieeexplore.ieee.org.

Digital Object Identifier 10.1109/TPWRS.2013.2278215 $q \in \mathcal{Q} \quad$ Model complexity levels $\mathcal{Q}=$ $\{$ low, med, high $\}$.

$\tilde{t} \in \tilde{\mathcal{T}} \quad$ Hours.

$t, \tau, t \in \mathcal{T}$ Time intervals (of variable duration).

$\mathcal{T}^{q} \subseteq \mathcal{T} \quad$ Subset of time intervals for which model complexity level $q$ applies.

$t \in \mathcal{T}^{-} \quad$ Time intervals extended to the past.

$t \in \mathcal{T}^{+} \quad$ Time intervals extended to the future.

$w \in \mathcal{W} \quad$ Wind farms.

$\varphi \in \Phi \quad$ Unit operating phases $\Phi=$ $\{$ syn, soak, disp, des\}: syn: synchronization, soak: soak, disp: dispatchable, des: desynchronization.

\section{B. Parameters}

$A_{i}^{b, c} \quad$ Shift factor of unit $i$ on branch $b$, during contingency $c$, in p.u.

$A_{\ell}^{b, c} \quad$ Shift factor of load $\ell$ on branch $b$, in contingency

$A_{w}^{b, c}$

$B_{i, f, t}$

$C_{i, f, t}$

$h_{t}$

$N L C_{i}$

$P_{b}^{\max , c}$

$P_{i}^{\max (\min )}$ $c$

Shift factor of wind farm $w$ on branch $b$, in contingency $c$.

Size of step $f$ of unit $i$ marginal cost function, during time interval $t$, in MW.

$P_{i}^{\max , A G C}$ Maximum power output of unit $i$ while operating under $\mathrm{AGC}$, in $\mathrm{MW}$.

$P_{i}^{\min , A G C} \quad$ Minimum power output of unit $i$ while operating under $\mathrm{AGC}$, in $\mathrm{MW}$.

$P_{i, \tau, t}^{s o a k, j} \quad$ Power output of unit $i$, at soak phase, during time interval $t$, after a $j$ type start-up that initiates at time interval $\tau$, in MW.

$P_{i, \tau, t}^{\text {des }} \quad$ Power output of unit $i$, at desynchronization phase, during time interval $t$, before the respective shut-down that occurs at time interval $\tau$, in MW. 
$P_{\ell, t} \quad$ Effective load $\ell(=$ system load - imports + exports + pumping), during time interval $t$, in MW.

$P_{w, t} \quad$ Available wind power in wind farm $w$, during time interval $t$, in MW.

$R_{i}^{\max , m} \quad$ Maximum contribution (ramp limited) of unit $i$ in reserve type $m$, in $\mathrm{MW}$.

$R D_{i} \quad$ Ramp-down rate of unit $i$, in $\mathrm{MW} / \mathrm{min}$.

$R_{t}^{s y s, m} \quad$ System requirement in reserve type $m$ during time interval $t$, in MW.

$R U_{i} \quad$ Ramp-up rate of unit $i$, in $\mathrm{MW} / \mathrm{min}$.

$S D C_{i} \quad$ Shut-down cost of unit $i$, in $€$.

$S U C_{i}^{j} \quad$ Start-up cost of unit $i$ from start-up type $j$ until load with synchronization, in $€$.

$T \quad$ Number of time intervals of the planning horizon.

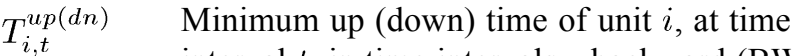
interval $t$, in time intervals - backward (BW) looking.

$T_{i, t}^{h w(w c)} \quad$ Off-load time before going from hot (warm) standby to warm (cold) standby condition of unit $i$, at time interval $t$, in time intervals - $\mathrm{BW}$ looking.

$T_{i, t}^{s y n, j} \quad$ Synchronization time of unit $i$ under start-up type $j$, at time interval $t$, in time intervals-BW looking.

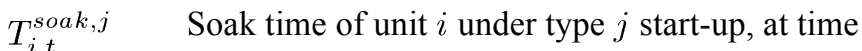
interval $t$, in time intervals-BW looking.

$T_{i, t}^{\text {des }} \quad$ Desynchronization time of unit $i$, at time interval $t$, in time intervals-BW looking.

\section{Variables}

\section{$\beta_{i, f, t}$}

Portion of step $f$ of the $i$ th unit's marginal cost function loaded in time interval $t$, in p.u.

$p_{i, t}$ Power output of unit $i$ during time interval $t$, in MW.

$p_{i, t}^{\text {des(soak) }} \quad$ Power output of unit $i$ during the desynchronization (soak) phase, at time interval $t$, in MW.

$p_{w, t} \quad$ Power output of wind farm $w$, at time interval $t$, in MW.

$r_{i, t}^{m} \quad$ Contribution of unit $i$ in reserve type $m$, during time interval $t$, in MW.

$u_{i, t} \quad$ Binary variable which is equal to 1 if unit $i$ is on.

$u_{i, t}^{\varphi}$

$u_{i, t}^{A G C}$

$u_{i, t}^{3 N S}$ $y_{i, t}^{j}$

Binary variable which is equal to 1 if a type- $j$ start-up of unit $i$ is initiated during time interval $t$.

$z_{i, t} \quad$ Binary variable which is equal to 1 if unit $i$ is shut-down during time interval $t$.

\section{General Remarks on Notation}

(a) Tilded time constants are expressed in hours.

(b) Time constants expressed in time intervals under a forward-looking logic (see Section II-B) are marked with a star, e.g.:

$\tilde{T}_{i}^{u p} \quad$ Minimum up time of unit $i$, in $\mathrm{h}$.

$T_{i, t}^{\text {des }}{ }^{*} \quad$ Desynchronization time of unit $i$, at time interval $t$, in time intervals - forward (FW) looking.

\section{E. Acronyms}

$A G C \quad$ Automatic generation control.

$C C G T$ Combined cycle gas turbine.

$C O P \quad$ Current operating plan.

$D A M \quad$ Day-ahead market.

$E D \quad$ Economic dispatch.

$L A E D \quad$ Look-ahead economic dispatch.

$L M P \quad$ Locational marginal price.

$L P \quad$ Linear programming.

MILP Mixed integer linear programming.

OCGT Open cycle gas turbine.

$R A C \quad$ Reliability assessment commitment.

$R T M \quad$ Real time market.

$S M P \quad$ System marginal price.

ST Steam turbine.

$U C \quad$ Unit commitment.

$V G \quad$ Variable generation.

\section{INTRODUCTION}

$\mathbf{T}$ HE short-term operations scheduling of power systems has been traditionally based on a two level - unit commitment/economic dispatch-hierarchy paradigm [1]: day-ahead unit commitment (UC) scheduling is first performed at around 12:00 noon of the day preceding the dispatch day in order to determine the commitment status of all dispatchable units during all the dispatch periods of the dispatch day, with an hourly time resolution. Real-time economic dispatch (ED) is performed every $5 \mathrm{~min}$ and determines the active power output of all committed dispatchable units (unit base-points), for the next 5-min interval. ${ }^{1}$ Current UC practice assumes deterministic knowledge (perfect forecast) of system conditions for the next day, typically load demand and component availability. Reserve requirements and " $\mathrm{N}-1$ " contingency analysis ensure a certain degree of robustness against forecast errors. Until

\footnotetext{
${ }^{1} \mathrm{~A}$ third level, automatic generation control (AGC), that corrects the power output of the units every few seconds to account for demand fluctuations within the 5-min dispatch period, is outside the scope of this paper and will not be further discussed.
} 
recently, the perfect forecast was a reasonable assumption since both the load demand and the availability of the conventional, dispatchable units could be fairly accurately forecasted, with component failures within the next day considered a rather rare event. When system conditions deviate substantially from forecasts, forward (day-ahead) or intra-day revised UC schedules are computed.

The two level hierarchy paradigm of the traditional power system short-term operation has been transferred to the design of many centrally organized wholesale electricity markets, such as the ISO/RTO markets in the United States, in the form of the two-settlement system [2] comprising a day-ahead forward market (DAM) with hourly dispatch period and a real-time market (RTM) with 5-min dispatch period, complemented with a forward or intraday reliability assessment commitment (RAC). RAC commits resources based on load forecasts and not solely on participant offers, bids and schedules used by DAM. Forward or intraday RACs adapt resource commitment to system condition changes.

The growth of variable (intermittent) renewable energy in the generation mix of many power systems has challenged the traditional paradigm of the short-term system operation [3]-[9]. Variable generation (VG) technologies deliver energy on an as-available basis, and increase the level of variability and uncertainty in power system operations. As the VG penetration further increases, current market and short-term operating practices will be inadequate and need to be revised. Advanced forecasting tools, especially for $\mathrm{VG}$, are required and stochastic/robust centralized scheduling tools will replace the traditional deterministic scheduling tools of today [10]-[18]. In addition, more frequently revised forward and intraday UC schedules and dispatch scheduling with sub-hourly dispatch intervals and look-ahead features are required. Centrally organized wholesale electricity markets of North America are implementing such changes in their operating practices [17]-[20]. In [17] following a day-ahead RAC, an hour-ahead RAC is solved for every hour of the operating day, with hourly granularity and scheduling horizon endpoint equal to the one of the last executed day-ahead RAC. The basic idea behind [18]-[20] is to implement a short-term (next 3-6 h) MILP-based fast-start unit commitment adjustment with sub-hourly (15-min, 30-min) dispatch periods and an LP-based look-ahead dispatch scheduling for the next hour with 5-min dispatch period, with appropriate, yet complex, interface between them. While responding to recently issued FERC Order 764 [21] some US markets plan to convert this short-term fast-start unit commitment to a financially binding market that bridges the gap between DAM and RTM [22]. The economic benefits of extending the horizon of the look-ahead dispatch in anticipation of steep variations of renewable generation, as well as the computational implications are analyzed in [23]. As pointed out in [18]-[20] inconsistencies in the modeling complexity and the time resolution of the various short-term scheduling models may create operational problems during highly dynamic environments.

This paper introduces the idea of unified UC-ED modeling within a unique tool that performs ED with up to 24-h look-ahead capability. Variable time resolution and variable

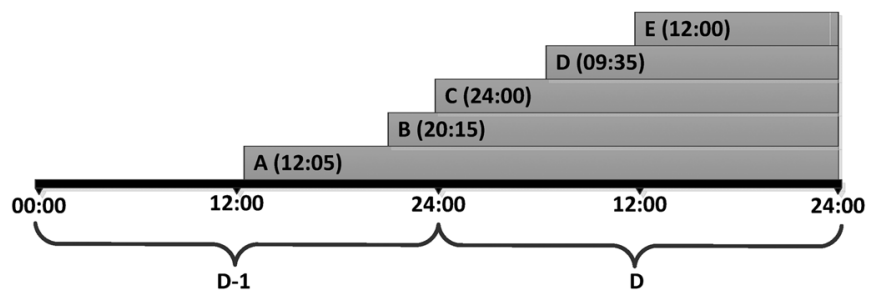

Fig. 1. Scheduling horizon definition.

modeling complexity are used in order to reduce the computational requirements of the model. A finer time resolution and detailed modeling are used during the first hours of the scheduling horizon while coarser time resolution and simplified modeling during the last ones. The proposed model combines the current look-ahead commitment and real-time dispatch functions, providing binding dispatch instructions (Base Points) for the next 5-min interval and anticipated power system operating conditions (unit commitment and dispatch) for the remaining scheduling horizon. It is an extension of the look-ahead commitment/dispatch model proposed in [18] and [19]. The advantages of the proposed model are the simplicity of maintaining a single short-term model, the avoidance of the interface between different models for different short-term time-scales and the smooth transition between UC and ED, controlled only by the initial conditions. The basic disadvantage, when applied to long look-ahead horizons, is its computational requirements. Currently it is applicable to medium-sized systems, as demonstrated by the test results. Advances in optimization algorithms and computer hardware are expected to make our model applicable to large power systems in the near future.

\section{Unified COMMitMent—Dispatch Model}

\section{A. Variable Time Resolution and Complexity Modeling}

To facilitate the presentation we begin with the description of a particular implementation of the variable time resolution and complexity unified UC-ED model, by specifying the scheduling horizon, the variable time resolution, the variable modeling complexity and the input requirements. The implementation (timelines, time steps, look-ahead horizon, etc.) is indicative and should be adapted to the particular power system.

1) Scheduling Horizon: Assuming that the DAM gate closure is 12:00 noon, the unified UC-ED scheduling horizon varies from a maximum of $36 \mathrm{~h}$ to a minimum of $12 \mathrm{~h}$ as shown in Fig. 1, where the numbers in parenthesis indicate the beginning (hh:mm) of the scheduling horizon in five different cases, A-E.

2) Variable Time Resolution: The basic idea of variable time resolution is to use a 5-min time step for the 1 st scheduling hour, a 15-min time step for the 2nd hour, a 30-min time step for the 3 rd hour, and hourly time step for the remaining scheduling horizon. This situation is shown in the second row of Table I where the scheduling begins at an exact clock hour. In order to align the variable time intervals with clock hours, in case the scheduling does not begin at an exact clock hour, the following 
TABLE I

DEFINITION OF THE VARIABLE TIME INTERVALS

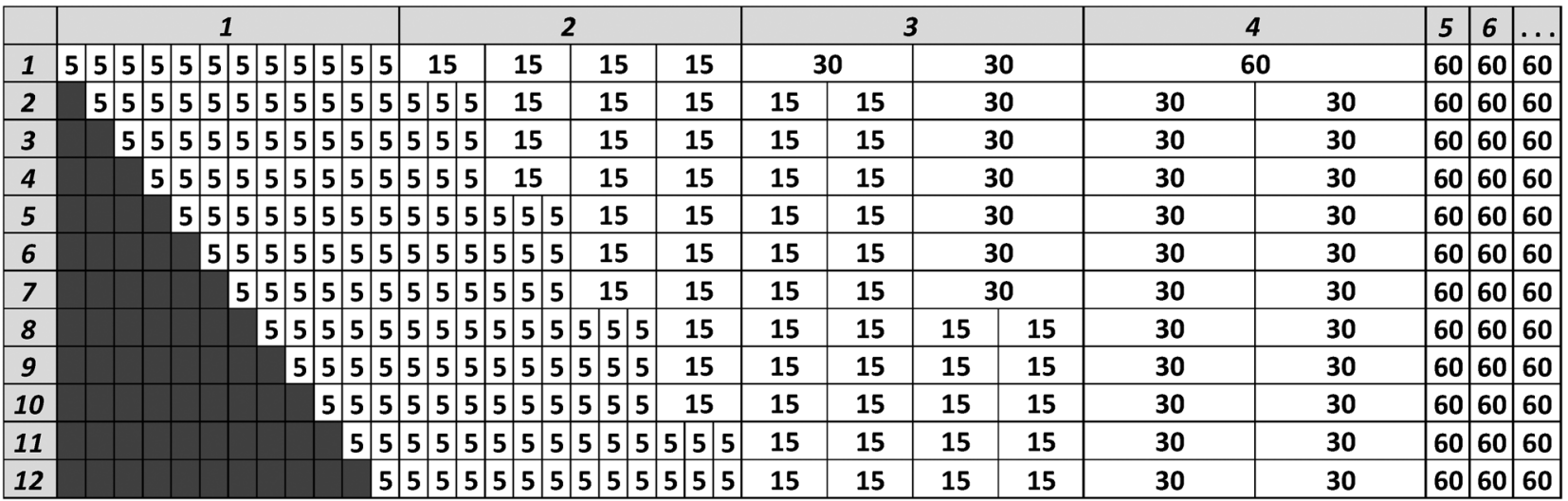

TABLE II

VARIABLE MODEL COMPLEXITY

\begin{tabular}{|c|c|c|}
\hline Time Step & Level & Modeling Complexity \\
\hline $5-\min$ & high & $\begin{array}{l}\text { Unit technical constraints } \\
\text { Power Balance \& Reserve Requirements } \\
\text { Full Network Representation: } \\
\text { Base Case and full set of Contingencies }\end{array}$ \\
\hline $\begin{array}{c}15-\min \& \\
30-\min \end{array}$ & med & $\begin{array}{c}\text { Unit technical constraints } \\
\text { Power Balance \& Reserve Requirements } \\
\text { Full Network Representation: } \\
\text { Base Case and reduced set of Contingencies }\end{array}$ \\
\hline $60-\min$ & low & $\begin{array}{c}\text { Unit technical constraints } \\
\text { Power Balance \& Reserve Requirements } \\
\text { Full Network Representation: } \\
\text { Base Case only }\end{array}$ \\
\hline
\end{tabular}

rules apply: 1) use a 5-min time step for at least one hour, followed by a 15-min time step for at least one hour, followed by a 30-min time step for at least one hour, and hourly time step thereafter; 2) align intervals of a specific duration with intervals of the immediately longer duration; 3) span the scheduling horizon with the minimum number of intervals. All possible cases are presented in Table I, where the column header denotes clock hour and the row header denotes the 5-min interval within the hour in which the scheduling begins.

Combining the information of Fig. 1 and Table $\mathrm{I}$ it is evident that the size of the planning horizon ranges from a minimum of 29 time intervals to a maximum 54 time intervals.

3) Variable Model Complexity: Table II presents the three different levels of modeling complexity used in our implementation. The modeling complexity is aligned with the time-step duration, so that a detailed UC model is used for the near future periods and a simplified model is used for the far future periods. The model complexity levels proposed in Table II are indicative and system specific. A linear DC network model is used in all complexity levels in our implementation.

An envisaged real-life application in advanced electricity markets would use a full AC network model with a full set of contingencies in the first hour of scheduling (5-min time step), a full DC network model with a reduced monitored contingency set for the next $2 \mathrm{~h}$ (15-min and 30-min time step) and a reduced DC network model with an even more reduced monitored contingency set for the remaining horizon (60-min time step). However, increasing modeling complexity increases computational requirements.

4) Input Requirements: The unified UC-ED is executed on a rolling basis every five minutes and provides binding dispatch instructions (Base-Points) and prices (LMPs) for the next 5-min time interval and anticipated power system operating conditions (commitment and dispatch) for the remaining scheduling horizon.

The application of the model requires the following key inputs: 1) real-time initial conditions and network data from the state estimator, 2) unit offer parameters (including technical data, such as ramp-rates, minimum up/down times, etc.) and 3) forecasts of the load demand and VG.

\section{B. Constraint Conversion to Variable Time Step}

Before presenting the mathematical formulation of the variable time step UC problem we first describe how the constraints of a constant (hourly) time step UC model, which closely follows [24], are converted to constraints of a variable time step UC model.

As stated in the Nomenclature Section, $\tilde{t}$ denotes time in hours, whereas $t$ denotes time intervals of variable duration. Similarly, UC time constants expressed in hours are tilded, e.g., $\tilde{T}_{i}^{u p}$.

Constraints (1) and (2) express the minimum-up-time constraint of a unit $i$ in constant (hourly) and variable time step model, respectively:

$$
\begin{array}{ll}
u_{i, \tilde{t}} \geq \sum_{\tilde{\tau}=\tilde{t}-\tilde{T}_{i}^{u p}+1}^{\tilde{t}} y_{i, \tilde{\tau}} & \forall \tilde{t} \in \tilde{\mathcal{T}} \\
u_{i, t} \geq \sum_{\tau=t-T_{i, t}^{u p}+1}^{t} y_{i, \tau} & \forall t \in \mathcal{T} .
\end{array}
$$

Both constraints ensure that unit $i$ is committed during hour $\tilde{t}$ if it was started up within the prior $\tilde{T}_{i}^{u p}$ hours. It is observed that both constraints are identical, except from the fact that the minimum up time expressed in intervals in (2) is indexed on time interval, $T_{i, t}^{u p}$.

This is explained with the help of Fig. 2 representing part of a variable time step scheduling horizon of a unit with $\tilde{T}_{i}^{u p}=4 \mathrm{~h}$. 


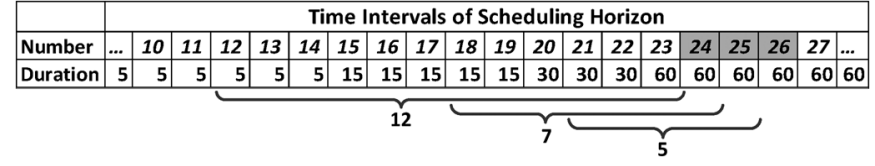

Fig. 2. Example that shows how minimum-up time of $\tilde{T}_{i}^{u p}=4 \mathrm{~h}$ is converted to be used in a variable time resolution modeling.

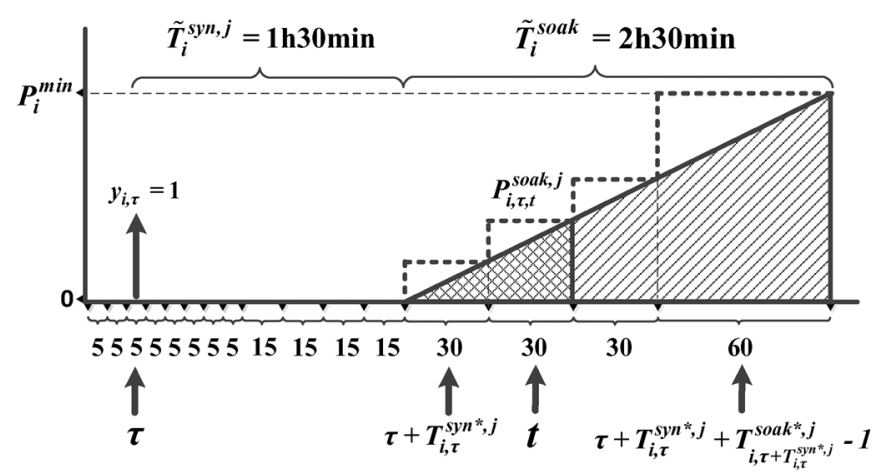

Time intervals' duration (min)

Fig. 3. Unit power output during soak (it is assumed that the unit is started up during interval $\tau$ ).

The 2nd row of Fig. 2 represents the time intervals, consecutively numbered, and the 3 rd row their duration in minutes. As shown in the figure, the arithmetic value of the minimum up time, $T_{i, t}^{u p}$, expressed in time intervals, depends on the time interval $t$ at which it terminates (for backward-looking modeling [24]) or at which it starts (for forward-looking modeling [24]). Thus, the minimum up time of 4 hours, corresponds to 12,7 or 5 time intervals depending on whether the terminating interval (in fact the one immediately following, in our modeling) is 24 , 25 or 26 , respectively. That is, in (2), which follows backwardlooking modeling, $T_{i, 24}^{u p}=12, T_{i, 25}^{u p}=7$ and $T_{i, 26}^{u p}=5$. Since certain constraints, such as the desynchronization constraints, follow forward-looking modeling [24], forward-looking time constants are also required and are denoted by an asterisk, such as $T_{i, t}^{u p^{*}}$. In the example of Fig. $2, T_{i, 12}^{u p^{*}}=12, T_{i, 18}^{u p^{*}}=7$ and $T_{i, 21}^{u p^{*}}=5$. Note that, in general, $T_{i, t}^{u p^{*}} \neq T_{i, t}^{u p}$.

Another point that requires clarification is the modeling of the unit power output during the soak and the desynchronization phases [24]. Assuming linear increase (decrease) of the unit power output from zero to technical minimum (vice versa) during the soak (desynchronization) phase, the power output during these two phases is defined by (3) and (4), respectively. Figs. 3 and 4 explain the derivation of (3) and (4) with the analysis of the two similar triangles (line and cross-shaded, respectively). For example in Fig. 3, which explains (3), one can identify four distinct time intervals: the start-up (beginning of the synchronization phase) interval $\tau$, the beginning of the soak phase interval $\tau+T_{i, \tau}^{s y n^{*}, j}$, the current time interval $t$, and the end of the soak phase interval $\tau+T_{i, \tau}^{s y n^{*}, j}+T_{i, \tau+T_{i,}^{s y n^{*}, j}}^{s o a k^{*}, j}-1$. The numerator of (3) is equal to the time elapsed from the beginning of the soak phase till the end of interval $t(30+30=60 \mathrm{~min})$; the denominator equals the total soak phase time $(30+30+$

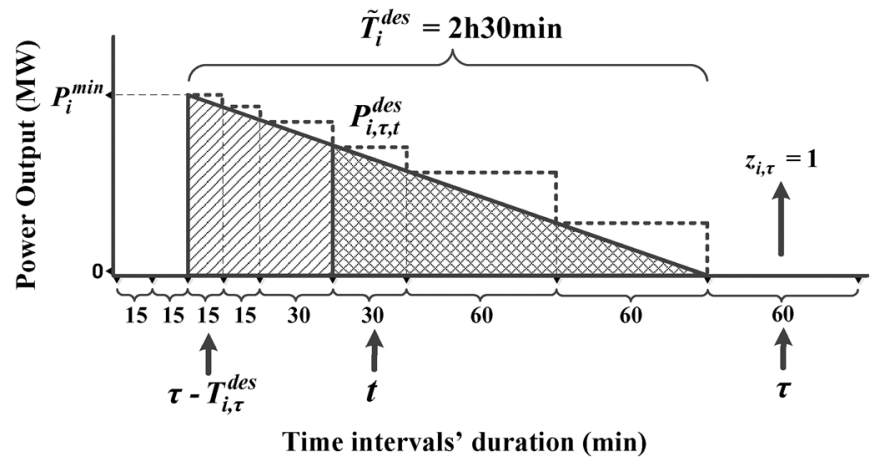

Fig. 4. Unit power output during desynchronization (it is assumed that the unit is shut down during interval $\tau$ ).

$30+60=150 \mathrm{~min})$. Note that time constants in Fig. 3 are forward looking (denoted by a star) while time constants in Fig. 4 are backward looking:

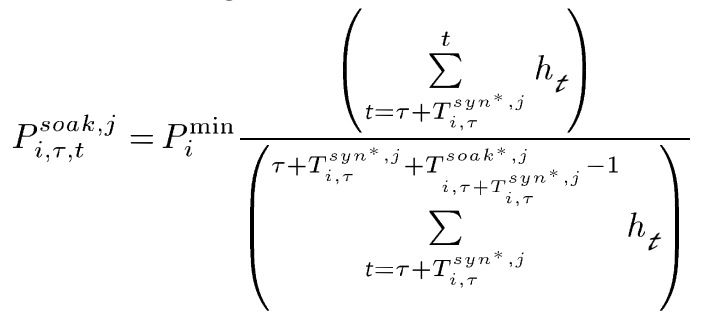

$$
\begin{aligned}
& \forall i \in I^{\mathcal{T} \mathcal{H}}, \tau \in \mathcal{T}^{-}, \tau+T_{i, \tau}^{s y n^{*}, j} \leq t \leq \tau \\
& +T_{i, \tau}^{s y n^{*}, j}+T_{i, \tau+T_{i, \tau}^{s y n^{*}, j}}^{s o a k^{*}, j}-1 \\
& P_{i, \tau, t}^{s o a k, j}=0 \quad \text { otherwise } \\
& P_{i, \tau, t}^{d e s}=\frac{P_{i}^{\min }\left(\sum_{t=t}^{\tau-1} h_{t}\right)}{\left(\sum_{t=\tau-T_{i, \tau}^{d \epsilon s}}^{\tau-1} h_{t}\right)} \quad \forall i \in I^{\mathcal{T} \mathcal{H}}, \tau \in \mathcal{T}^{+}, \\
& \tau-T_{i, \tau}^{\text {des }} \leq t \leq \tau-1 \\
& P_{i, \tau, t}^{\text {des }}=0 \quad \text { otherwise. }
\end{aligned}
$$

\section{Mathematical Formulation of the Variable Time-Step UC}

The variable time-step UC is formulated as an MILP optimization problem, as follows:

$$
\text { Minimize Cost }
$$

subject to the following set of constraints:

1) Cost Definition Equations:

$$
\begin{array}{r}
\text { Cost }=\sum_{t \in \mathcal{T}} \sum_{i \in I}\left(h _ { t } \cdot \left(\sum_{f \in \mathcal{F}^{i}}\left(C_{i, f, t} B_{i, f, t} \beta_{i, f, t}\right)+N L C_{i}\right.\right. \\
\left.\cdot\left(u_{i, t}-u_{i, t}^{s y n}\right)\right) \\
\left.+\sum_{j \in \mathcal{J}} S U C_{i}^{j} \cdot y_{i, t}^{j}+S D C_{i} \cdot z_{i, t}\right)
\end{array}
$$




$$
\begin{aligned}
& \sum_{f \in \mathcal{F}^{i}} B_{i, f, t} \cdot \beta_{i, f, t}=p_{i, t} \quad \forall i \in I, t \in \mathcal{T} \\
& 0 \leq \beta_{i, f, t} \leq 1 \quad \forall i \in I, f \in \mathcal{F}^{i}, t \in \mathcal{T} .
\end{aligned}
$$

Equality (6) defines the Cost as a function of the units' stepwise marginal cost function, no-load-cost, start-up and shutdown cost. Constraints (7) and (8) define the power output of the unit as a function of the variables $\beta$, which express the portion of the step $f$ of the unit's marginal cost function loaded during interval $t$.

2) Logical State of Commitment:

$$
\begin{array}{ll}
u_{i, t}=u_{i, t}^{s y n}+u_{i, t}^{\text {soak }}+u_{i, t}^{\text {disp }}+u_{i, t}^{\text {des }} & \forall i \in I, t \in \mathcal{T} \\
y_{i, t}-z_{i, t}=u_{i, t}-u_{i, t-1} & \forall i \in I, t \in \mathcal{T} \\
y_{i, t}+z_{i, t} \leq 1 & \forall i \in I, t \in \mathcal{T} .
\end{array}
$$

Constraints (9) ensure that if unit $i$ is online, only one of the commitment states is allowed. Constraints (10) relate the start-up and shut-down status of unit $i$, with its commitment status. Constraints (11) ensure that start-up and shut-down do not coincide.

\section{3) Start-Up Type Constraints:}

$$
\begin{array}{ll}
y_{i, t}=\sum_{j \in \mathcal{J}} y_{i, t}^{j} & \forall i \in I^{\mathcal{T H}}, t \in \mathcal{T} \\
y_{i, t}^{h} \leq \sum_{\tau=t-T_{i, t}^{h w}+1}^{t} z_{i, \tau} & \forall i \in I^{\mathcal{T H}}, t \in \mathcal{T} \\
y_{i, t}^{w} \leq \sum_{\tau=t-T_{i, t}^{w c}+1}^{t-T_{i, t}^{h w}} z_{i, \tau} & \forall i \in I^{\mathcal{T H}}, t \in \mathcal{T} \\
y_{i, t}^{c} \leq \sum_{\tau=1}^{t-T_{i, t}^{w}} z_{i, \tau} & \forall i \in I^{\mathcal{T H}}, t \in \mathcal{T} .
\end{array}
$$

Constraints (12) ensure that only one start-up type is allowed. Constraints (13) prohibit hot start-up of unit $i$ at time interval $t$ if it did not shut down in the prior $T_{i, t}^{h w}$ intervals. Similarly, constraints (14) and (15) prohibit warm or cold start-up of unit $i$ at time interval $t$, if it did not shut down in the appropriate time intervals prior to $t$.

4) Synchronization Phase Constraint:

$$
u_{i, t}^{\text {syn }}=\sum_{j \in \mathcal{J}}\left(\sum_{\tau=t-T_{i, t}^{s y n, j}+1}^{t} y_{i, \tau}^{j}\right) \forall i \in I^{\mathcal{T H}}, t \in \mathcal{T} \text {. }
$$

Constraint (16) ensures that unit $i$ is in synchronization phase during time interval $t$, if it incurred any type $j$ start-up during the prior $T_{i, t}^{s y n, j}$ intervals.

5) Soak Phase Constraints:

$$
\begin{aligned}
& u_{i, t}^{\text {soak }}=\sum_{j \in \mathcal{J}}\left(\sum_{\tau=t-T_{i, t}^{s o a k, j}-T_{i, t-T_{i, t}^{s y n}, j}^{\text {soa }, j}}^{t-T_{i, t}^{s y n, j}} y_{i, \tau}^{j}\right. \\
& \forall i \in I^{\mathcal{T H}}, t \in \mathcal{T}
\end{aligned}
$$

$$
\begin{gathered}
p_{i, t}^{\text {soak }}=\sum_{j \in \mathcal{J}}\left(\sum_{\tau=t-T_{i, t}^{s, a k, j}-T_{i, t-T_{i, t}^{s y n, j}}^{s o a k, j}}^{t-T_{i, t}^{s y n, j}} y_{i, \tau}^{j} \cdot P_{i, \tau, t}^{s o a k, j}\right) \\
\forall i \in I^{\mathcal{T H}}, t \in \mathcal{T} .
\end{gathered}
$$

Constraint (17) ensures that unit $i$ is in soak phase during interval $t$, if it incurred any type $j$ start-up during the period ranging from $t-T_{i, t}^{s o a k, j}-T_{i, t-T_{i, t}^{s o a k, j}}^{s y n, j}+1$ intervals before $t$ to $t-T_{i, t}^{s y n, j}$ intervals before $t$. Constraint (18) determines the power output of unit $i$, during the soak phase in terms of $P_{i, \tau, t}^{\text {soak }, j}$ defined in (3). Note that the summation limits of (17) and (18) represent hourly values $\tilde{t}-\tilde{T}_{i}^{\text {soak }, j}-\tilde{T}_{i}^{s y n, j}+1$ and $\tilde{t}-\tilde{T}_{i}^{\text {soak,j }}$ respectively [24] converted to time intervals using backward looking logic. A similar conversion using forward looking logic was explained with the help of Fig. 3.

6) Desynchronization Phase Constraints:

$$
\begin{aligned}
u_{i, t}^{\text {des }} & =\sum_{\tau=t+1}^{t+T_{i, t+1}^{\text {des* }}} z_{i, \tau} & \forall i \in I^{\mathcal{T H}}, t \in \mathcal{T} \\
p_{i, t}^{\text {des }} & =\sum_{\tau=t+1}^{t+T_{i, t+1}^{\text {des* }}} z_{i, \tau} \cdot P_{i, \tau, t}^{\text {des }} & \forall i \in I^{\mathcal{T H}}, t \in \mathcal{T} .
\end{aligned}
$$

Constraint (19) ensures that unit $i$ is in desynchronization phase during interval $t$, if it shuts down in the next $T_{i, t+1}^{d e s^{*}}$ intervals. Constraint (20) determines the power output of unit $i$, during the desynchronization phase, in terms of $P_{i, \tau, t}^{d e s}$ defined in (4).

7) Minimum Up/Down Constraints:

$$
\begin{aligned}
u_{i, t} & \geq \sum_{\tau=t-T_{i, t}^{u p}+1}^{t} y_{i, \tau} \quad \forall i \in I^{\mathcal{T H}}, t \in \mathcal{T} \\
1-u_{i, t} & \geq \sum_{\tau=t-T_{i, t}^{d n}+1}^{t} z_{i, \tau} \quad \forall i \in I^{\mathcal{T H}}, t \in \mathcal{T} .
\end{aligned}
$$

Constraint (21) ensures that unit $i$ remains online during interval $t$, if it started-up within the previous $T_{i, t}^{u p}$ intervals. Constraint (22) ensures that unit $i$ remains offline if it was shut down within the previous $T_{i, t}^{d n}$ intervals.

8) Ramp Up/Down Constraints:

$$
\begin{aligned}
p_{i, t}-p_{i, t-1} \leq & R U_{i} \cdot h_{t-1}+P_{i}^{\max } \\
& \cdot\left(y_{i, t}+u_{i, t}^{\text {syn }}+u_{i, t}^{\text {soak }}\right) \quad \forall i \in I, t \in \mathcal{T} \\
p_{i, t-1}-p_{i, t} \leq & R D_{i} \cdot h_{t-1}+P_{i}^{\max } \\
& \cdot\left(u_{i, t-1}^{\text {des }}+z_{i, t}\right) \quad \forall i \in I, t \in \mathcal{T} .
\end{aligned}
$$

Constraints (23) and (24) enforce the unit ramp-rate limits. The last terms of both constraints relax the ramp-rate limits during synchronization, soak and desynchronization phase.

9) Power Output Constraints:

$$
\begin{gathered}
p_{i, t}+r_{i, t}^{1}+r_{i, t}^{2+}+r_{i, t}^{3 S} \\
\leq 0 \cdot u_{i, t}^{s y n}+p_{i, t}^{\text {soak }}+p_{i, t}^{\text {des }}+P_{i}^{\max } \cdot u_{i, t}^{\text {disp }} \\
\forall i \in I, t \in \mathcal{T}
\end{gathered}
$$




$$
\begin{aligned}
& p_{i, t}-r_{i t}^{1-}-r_{i t}^{2-} \\
& \geq 0 \cdot u_{i, t}^{s y n}+p_{i, t}^{s o a k}+p_{i, t}^{d e s}+P_{i}^{\min } \cdot u_{i, t}^{d i s p} \\
& \forall i \in I, t \in \mathcal{T} \\
& p_{i, t}+r_{i t}^{2+} \\
& \leq 0 \cdot u_{i, t}^{\text {syn }}+p_{i, t}^{\text {soak }}+p_{i, t}^{\text {des }}+P_{i}^{\max } \cdot\left(u_{i, t}^{\text {disp }}-u_{i, t}^{A G C}\right) \\
& +P_{i}^{\max , A G C} \cdot u_{i, t}^{A G C} \quad \forall i \in I, t \in \mathcal{T} \\
& p_{i, t}-r_{i, t}^{2-} \\
& \geq 0 \cdot u_{i, t}^{s y n}+p_{i, t}^{s o a k}+p_{i, t}^{d e s}+P_{i}^{\min } \cdot\left(u_{i, t}^{d i s p}-u_{i, t}^{A G C}\right) \\
& +P_{i}^{\min , A G C} \cdot u_{i, t}^{A G C} \quad \forall i \in I, t \in \mathcal{T} .
\end{aligned}
$$

Constrains (25)-(28) constrain the unit power output and reserve commitment within the unit capabilities. The first three terms of the right hand side of the equations constrain the power output during synchronization, soak and desynchronization phase with the help of (18) and (20).

\section{0) Reserve Constraints:}

$$
\begin{array}{lr}
u_{i, t}^{A G C} \leq u_{i, t}^{\text {disp }} & \forall i \in I, t \in \mathcal{T} \\
0 \leq r_{i, t}^{m} \leq R_{i}^{\max , m} \cdot u_{i, t}^{d i s p} \quad \forall i \in I, t \in \mathcal{T}, m \in\left\{1^{+}, 1^{-}, 3 S\right\} & \\
0 \leq r_{i, t}^{m} \leq R_{i}^{\max , m} \cdot u_{i t}^{A G C} \quad \forall i \in I, t \in \mathcal{T}, m \in\left\{2^{+}, 2^{-}\right\} & \\
P_{i}^{\min } \cdot u_{i, t}^{3 N S} \leq r_{i, t}^{3 N S} \leq R_{i}^{\max , 3 N S} \cdot u_{i, t}^{3 N S} \quad \forall i \in I, t \in \mathcal{T} \\
u_{i, t}^{3 N S} \leq 1-u_{i, t} \quad \forall i \in I, t \in \mathcal{T} \\
\sum_{i \in I} r_{i, t}^{m} \geq R_{t}^{s y s, m} \quad \forall t \in \mathcal{T}, m \in\left\{1^{+}, 1^{-}, 2^{+}, 2^{-}\right\} \\
\sum_{i \in I} r_{i, t}^{3 S}+\sum_{i \in I} r_{i, t}^{3 N S} \geq R_{t}^{s y s, 3} \quad \forall t \in \mathcal{T} .
\end{array}
$$

Constraints (29)-(33) define the (ramp-limited) maximum contribution of each unit to the system reserves, for each reserve type. Note that a unit contributes to primary and tertiary spinning reserve only when in dispatchable phase, to secondary reserve only if it operates under AGC and to tertiary non-spinning reserve only when it is offline. Constraints (34) and (35) ensure that the total system reserve requirement is met, for all reserve types.

\section{1) Power Balance Equation:}

$$
\sum_{i \in I} p_{i, t}+\sum_{w \in \mathcal{W}} p_{w, t}=\sum_{\ell \in \mathcal{L}} P_{\ell, t} \forall t \in \mathcal{T}
$$

Constraint (36) ensures that the system power demand is matched by power supply in all time intervals.

12) Wind Power Constraints:

$$
p_{w, t} \leq P_{w, t} \quad \forall t \in \mathcal{T}, w \in \mathcal{W} .
$$

Constraint (37) ensures that wind power output of any wind farm does not exceed the available wind power.

13) Network Security Constraints:

$$
\begin{aligned}
-P_{b}^{\max , c} & \leq \sum_{i \in I} A_{i}^{b, c} \cdot p_{i, t}+\sum_{w \in \mathcal{W}} A_{w}^{b, c} \cdot p_{w, t}-\sum_{\ell \in \mathcal{L}} A_{\ell}^{b, c} \cdot P_{\ell, t} \\
& \leq P_{b}^{\max , c} \quad t \in \mathcal{T}^{q}, b \in \mathcal{B}^{c}, c \in C^{q}, q \in \mathcal{Q}
\end{aligned}
$$

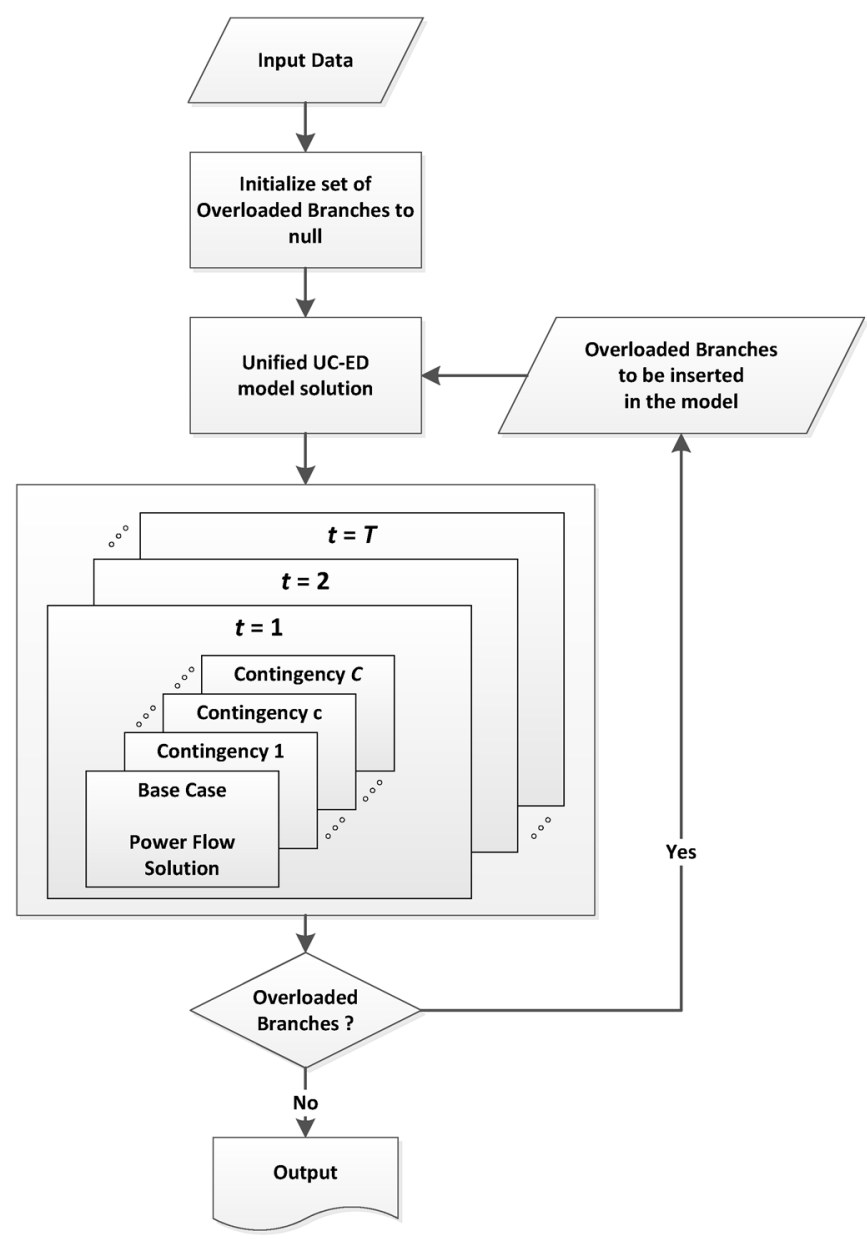

Fig. 5. Iterative algorithm that enforces network security constraints.

Constraints (38) enforce transmission limits, for both base case $(c=0)$ and contingencies $(c \neq 0)$, depending on the model complexity, $q$, valid in the time interval under consideration(39).

Network security constraints are enforced through an iterative algorithm as shown in Fig. 5. As shown in Fig. 5 a variable time-step unified UC-ED is first solved, neglecting transmission constraints. Next for each time-step, $t=1, \ldots, T$, network security is analyzed through a series of power flow solutions (base-case and contingencies). If at any time-step one or more branches are overloaded (in the base-case or in any contingent-case) a corresponding branch loading constraint (38) is added to the unified UC-ED model of the next iteration. The iterative process terminates once no overloads are observed in any time-step.

The set of contingencies analyzed at each time step depends on the time step duration as indicated in Table II and (39): The complete set of network topology instances (contingencies), $q=$ "high", is analyzed in the early stages of the scheduling horizon, as shown in Fig. 5 for $t=1$, whereas a reduced set of network topology instances is analyzed at later stages.

\section{Test Results}

The developed unified UC-ED method has been tested on the Greek Power System for two consecutive days, Monday 6 and Tuesday 7 February 2012. 
TABLE III

Summary of the GreEK GENERATION System DATA

\begin{tabular}{|c|c|c|c|c|}
\hline Fuel & Technology & Number & $\begin{array}{c}\text { Capacity } \\
(\mathrm{MW})\end{array}$ & $\begin{array}{c}\text { Marginal Cost } \\
\text { Range }(€ / \mathrm{MWh})\end{array}$ \\
\hline Lignite & ST & 18 & 4448 & $33.82-40.88$ \\
\hline \multirow{3}{*}{ Gas } & CCGT & 10 & 3991 & $83.25-150.00$ \\
\cline { 2 - 5 } & OCGT & 3 & 147 & $150.00-150.00$ \\
\cline { 2 - 5 } & ST & 2 & 339 & $108.00-111.00$ \\
\hline \multirow{2}{*}{ Oil } & ST & 4 & 698 & $107.67-115.00$ \\
\hline \multirow{2}{*}{ Hydro } & Gen. only & 13 & 2341 & $95.00-96.00$ \\
\cline { 2 - 5 } & Pumping & 4 & 693 & $95.50-95.95$ \\
\hline
\end{tabular}

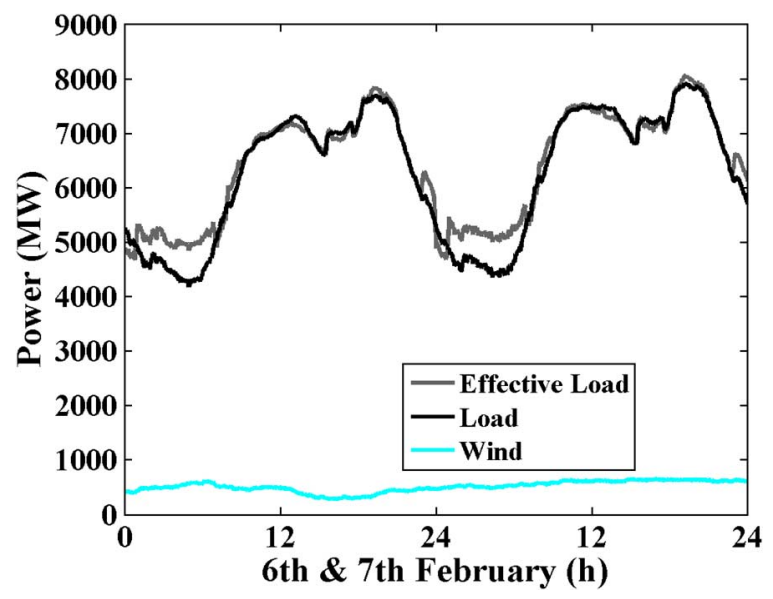

Fig. 6. System load and wind production for February 6 and 7, 2012.

TABLE IV

LoAd AND Wind Production Data (February 6 AND 7, 2012)

\begin{tabular}{|c|c|c|c|c|c|c|c|c|}
\hline \multirow[b]{2}{*}{ \& } & \multicolumn{4}{|c|}{ Load } & \multicolumn{4}{|c|}{ Wind } \\
\hline & $\begin{array}{c}\text { Max } \\
\text { (GW) }\end{array}$ & $\begin{array}{c}\text { Min } \\
(\mathrm{GW})\end{array}$ & $\begin{array}{l}\text { Mean } \\
\text { (GW) }\end{array}$ & $\begin{array}{l}\text { Energy } \\
\text { (GWh) }\end{array}$ & $\begin{array}{c}\text { Max } \\
\text { (GW) }\end{array}$ & $\begin{array}{l}\text { Min } \\
(\mathrm{GW})\end{array}$ & $\begin{array}{l}\text { Mean } \\
\text { (GW) }\end{array}$ & $\begin{array}{l}\text { Energy } \\
\text { (GWh) }\end{array}$ \\
\hline 6 & 7.36 & 4.25 & 6.04 & 145.0 & 0.83 & 0.298 & 0.530 & 12.73 \\
\hline 7 & 7.70 & 4.18 & 6.14 & 147.3 & 0.62 & 0.280 & 0.442 & 10.61 \\
\hline
\end{tabular}

The installed thermal and hydro capacity of the Greek power system is $12.5 \mathrm{GW}$, the installed wind capacity is $1.35 \mathrm{GW}$ and the peak load is about $10 \mathrm{GW}$. The Greek Power System comprises 1041 nodes, 54 units and 1286 high voltage $(150 \mathrm{kV}$ and $400 \mathrm{kV}$ ) branches. The generation system data are summarized in Table III.

Load and wind production data of February 6 and 7 are shown Fig. 6 and summarized in Table IV. One-minute historic data from the Historic Information System (HIS) of the Greek Independent Power Transmission Operator (IPTO) SCADA/EMS have been employed.

Three cases are examined for three different planning horizons, including the longest and shortest horizons (54 and 28 intervals) as shown in Table V. In the "high" model complexity, the next contingency set, against which the $\mathrm{N}-1$ system security is assessed, comprises all $58400-\mathrm{kV}$ branches of the Greek Power System. The next contingency set of the "med" model complexity comprises 20 critical branch outages. No contingencies are analyzed in the "low" model complexity (Table II).

The unified UC-ED problem was modeled in MATLAB R2012a [25] software using GAMS version 24.0.2 [26] along
TABLE V

TEST CASES DEscription

\begin{tabular}{|c|c|c|c|c|}
\hline & \multicolumn{4}{|c|}{ Planning Horizon } \\
\hline Case & Start & End & $\begin{array}{c}\text { Numb. of } \\
\text { Intervals }\end{array}$ & $\begin{array}{c}\text { Number of } \\
\text { Hours }\end{array}$ \\
\hline 1 & Oct-6; $12: 05$ & Oct-7; $24: 00$ & 54 & $\sim 36$ \\
\hline 2 & Oct-6; $19: 45$ & Oct-7; 24:00 & 44 & $\sim 30$ \\
\hline 3 & Oct-7; $11: 55$ & Oct-7; $24: 00$ & 28 & $\sim 12$ \\
\hline
\end{tabular}

TABLE VI

Optimization Model Size ANd EXeCUTION Time

\begin{tabular}{|c|c|c|c|c|c|c|c|c|}
\hline ర్ & & 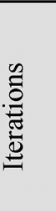 & 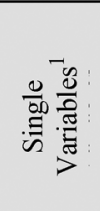 & 胥 & 莞 & 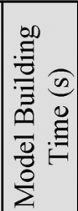 & 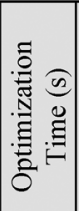 & 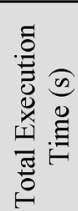 \\
\hline \multirow{2}{*}{1} & \multirow{2}{*}{2} & $1^{\mathrm{st}}$ & 128,331 & 31,174 & 78,772 & 6 & 11 & \multirow{2}{*}{73} \\
\hline & & $2^{\text {nd }}$ & 128,331 & 31,174 & 79,142 & 7 & 14 & \\
\hline \multirow{2}{*}{2} & \multirow{2}{*}{2} & $1^{\mathrm{st}}$ & 111,261 & 25,765 & 64,192 & 5 & 7 & \multirow{2}{*}{64} \\
\hline & & $2^{\text {nd }}$ & 111,261 & 25,765 & 66,888 & 8 & 9 & \\
\hline \multirow{2}{*}{3} & \multirow{2}{*}{2} & $1^{\mathrm{st}}$ & 83,949 & 17,062 & 40,864 & 4 & 3 & \multirow{2}{*}{54} \\
\hline & & $2^{\text {nd }}$ & 83,949 & 17,062 & 44,734 & 8 & 5 & \\
\hline
\end{tabular}

with CPLEX version 12.5 [27] solver. MATLAB is used for data input, power flow and shift factor calculations while GAMS is used for the solution of the MIP problem. A relative optimality gap of $0.05 \%$ was used. All tests were performed on a 3.5-GHz Intel Quad Core processor with 16 GB of RAM, running 64-bit Windows. Table VI summarizes the computational complexity of the models of the three cases of Table $\mathrm{V}$, presenting the number of single variables, integer variables and equations, as well as the model building time, optimization time and total simulation time. The difference between the total simulation time and the sum of the model building and optimization times (over all iterations) represents the time required for the solution of the load flows (base-case and contingencies) and the calculation of the shift factors in MATLAB. This time (about $35 \mathrm{~s}$ ) can be drastically reduced by parallel processing of the load flow solutions over time and over contingencies.

Figs. 7 and 8 present the aggregate power output of each generation technology and the system marginal price (SMP) for all 28 time intervals of Case 3. The SMP is defined as the LMP of the slack node located at the Greece-Bulgaria border.

In Case 3, one $150-\mathrm{kV}$ line is overloaded and two iterations are needed to redispatch/recommit the units (Fig. 5) in order to manage congestion. Table VII presents the capacity value of this $150-\mathrm{kV}$ line and the LMP range for all time intervals. When the line capacity value is zero, all buses have the same LMP which is equal to the LMP of the slack bus (Figs. 7 and 8).

Another case, Case 4, is studied to assess the response of our method to a sudden wind production change in comparison to the traditional separate UC-ED scheduling. To this purpose a sudden, trapezoidal-shaped change in wind production is introduced between 04:00 and 05:00 of February 6, as follows: wind production ramps up from 0 to $500 \mathrm{MW}$ within $20 \mathrm{~min}$, remains constant at $500 \mathrm{MW}$ for the next $20 \mathrm{~min}$ and ramps down to 0 in the last $20 \mathrm{~min}$. The total energy of the wind production change 


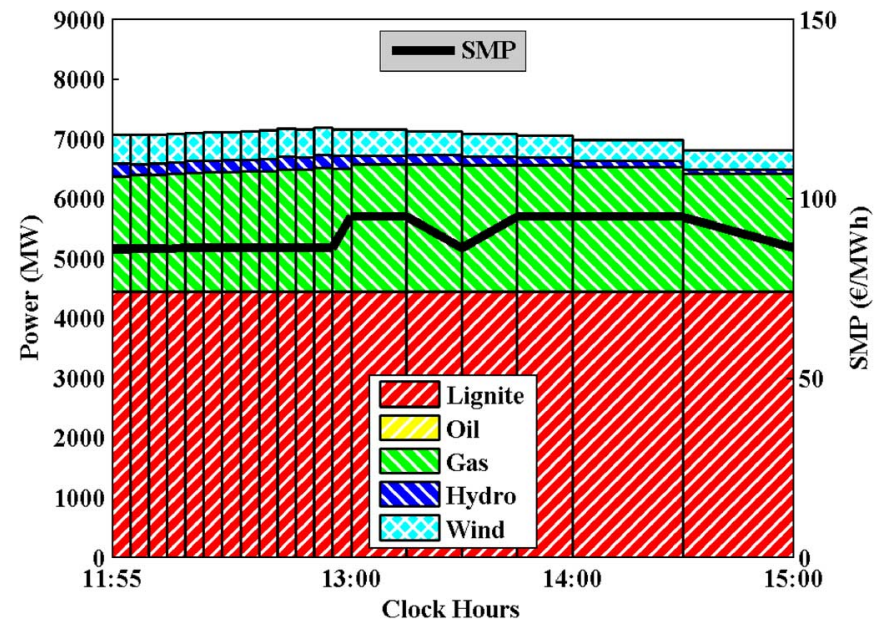

Fig. 7. Generation mix and SMP for 5-min, 15-min, and 30-min time intervals.

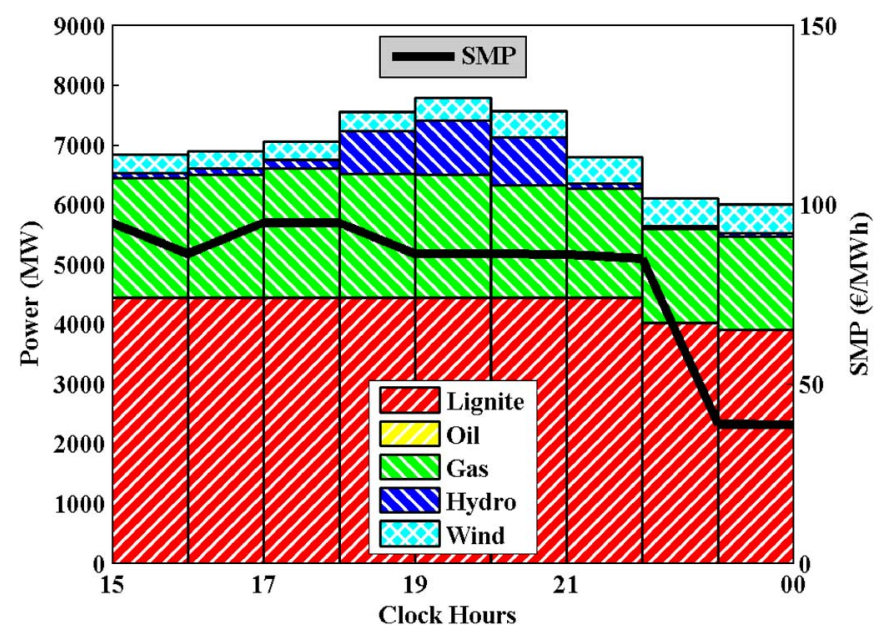

Fig. 8. Generation mix and SMP for 60 -min time intervals.

TABLE VII

CAPACITY VALUe OF THE 150-kV LiNE AND LMP RANGe

\begin{tabular}{|c|c|c|c|r|}
\hline $\begin{array}{c}\text { Time } \\
\text { Interval }\end{array}$ & $\begin{array}{c}\text { From-To } \\
\text { Time }\end{array}$ & $\begin{array}{c}\text { Line Capacity } \\
\text { Value }(€ / M W h)\end{array}$ & $\begin{array}{c}\text { Min LMP } \\
(€ / M W h)\end{array}$ & $\begin{array}{c}\text { Max LMP } \\
(€ / M W h)\end{array}$ \\
\hline 1 & $11: 55-12: 00$ & 191,91 & 82,60 & 221,85 \\
\hline $2-4$ & $12: 00-12: 15$ & 191,76 & 82,61 & 221,75 \\
\hline $5-13$ & $12: 15-13: 00$ & 184,64 & 83,10 & 217,07 \\
\hline $14-15$ & $13: 00-13: 30$ & 17,85 & 94,53 & 107,48 \\
\hline $16-21$ & $13: 30-17: 00$ & 184,64 & 83,10 & 217,07 \\
\hline 22 & $17: 00-18: 00$ & 17,85 & 94,53 & 107,48 \\
\hline $23-24$ & $18: 00-20: 00$ & 185,60 & 83,08 & 217,75 \\
\hline 25 & $20: 00-21: 00$ & 190,82 & 82,73 & 221,18 \\
\hline 26 & $21: 00-22: 00$ & 0,00 & 85,55 & 85,55 \\
\hline 27 & $22: 00-23: 00$ & 0,00 & 38,66 & 38,66 \\
\hline 28 & $23: 00-24: 00$ & 0,00 & 38,57 & 38,57 \\
\hline
\end{tabular}

is $341.67 \mathrm{MWh}$. Owing to the fact that the sudden increase of wind production occurs during the low load period (Fig. 6) when most thermal units operate close to their minimum power output, wind spillage may be required in order to satisfy the unit minimum power output constraints. Our unified UC-ED scheduling (Case $4 \mathrm{~A})$ is executed on a rolling basis every five minutes, continuously adapting both unit commitment and economic dis-

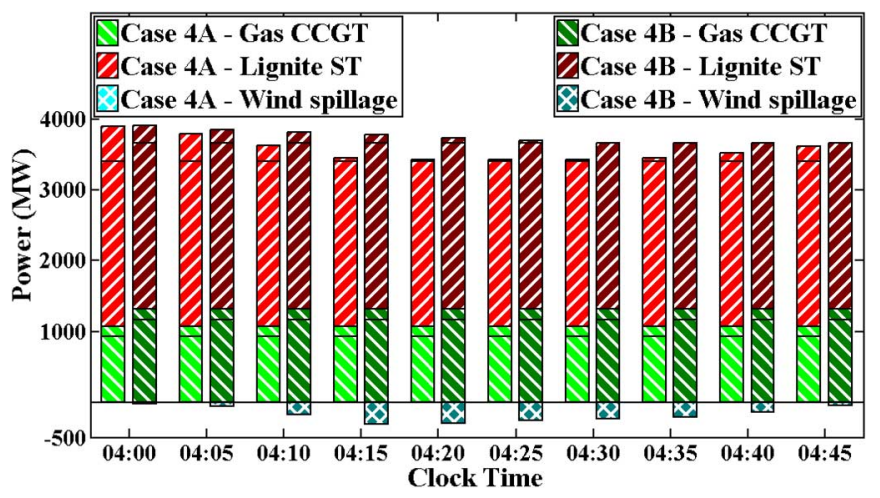

Fig. 9. CCGT and lignite-unit output and wind spillage during wind ramp-up.

patch to system condition changes. It is assumed that the sudden wind production change is predicted $15 \mathrm{~min}$ in advance.

The traditional UC-ED scheduling (Case 4B) executes a UC in the beginning of each day and keeps the unit commitment of the slow units fixed. Based on the fixed unit commitment an ED is performed every five minutes, without look-ahead capability. When real-time conditions deviate substantially from forecasts and the ED is infeasible, start-up or shut-down of fastresponding units (hydro or OCGT) is triggered.

Case 4C (UC-LAED) is identical to Case 4B except from the fact that the economic dispatch has 60-min look-ahead capability (LAED): it solves a linear program (LP) which simultaneously optimizes the next twelve 5-min time intervals, based on updated hour-ahead forecasts.

All three scheduling methods start with the same initial conditions on February 5, 24:00 am. No wind spillage was necessary in our unified UC-ED scheduling, which was able to adapt to the sudden change owing to its look-ahead capability. The traditional UC-ED scheduling results in wind spillage during the hours of the sudden wind increase. Owing to the technical minima constraints of thermal units and the lack of intraday look-ahead capability, $44 \%$ of the sudden wind power increase was spilled. Despite its look-ahead capability, the UC-LAED scheduling resulted in $33 \%$ wind curtailment, owing to the fact that it did not update the UC on a rolling basis.

Fig. 9 presents the power output of lignite-fired and gas-fired CCGT units during hours 04:00-04:45 of February 6 for cases $4 \mathrm{~A}$ and $4 \mathrm{~B}$. It is evident that our method has secured higher ramp-down capability in order to respond to the sudden wind increase during the low load hours. This is due to the fact that a 390-MW CCGT unit and a 30-MW lignite unit are not committed in Case 4A and the lignite-fired units operate at a safer distance from their technical minima relative to Case 4B: the horizontal lines in Fig. 9 separate the total power output of a particular technology in two parts: The lower part is the sum of the technical minima of all committed units of the respective technology and is the lower bound below which the total power output of the units of the particular technology cannot be further reduced. The upper part is the "flexible", above technical minima, part of the power output that can be reduced down to the horizontal line in order to counter-balance the sudden increase of wind power. It is evident that the wind spillage ob- 
served in Case 4B is equal to the ramp-down deficiency of Case $4 \mathrm{~B}$ relative to Case 4A.

\section{DisCUSSION ON IMPLEMENTATION ISSUES}

So far in our modeling and test results it has been assumed that the unified UC-ED scheduling is executed on a rolling basis every five minutes, continuously adapting both unit commitment and economic dispatch of all units to system condition changes. In reality, the commitment status of some long start units (in our case large lignite, gas and oil steam units) is decided in the day ahead market, while there may be some extra-long start units whose commitment status is decided using a multi-day RAC run [28]. Our model can be easily adapted to include commitment constraints on long start and extra-long start units, of the form

$$
u_{i, t} \geq u_{i, t}^{C O P} \quad \forall i \in I^{\mathcal{L S}}, \forall t \in \mathcal{T}
$$

where $u_{i, t}^{C O P}$ is the unit commitment status in the current operating plan (COP) and $I^{\mathcal{L S}}$ is the set of the long start units (which will also include the extra-long start units in the sequel). The current operating plan of all long start units is initialized to the unit commitment of the DAM and is updated every five minutes on a rolling basis. Thus, once a long start unit is committed either at initialization or at some stage of the unified UC-ED run, it cannot be de-committed at a subsequent UC-ED run. The inclusion of constraints (40) stabilizes the commitment of long start units, while allowing for the commitment of additional long start units for reliability purposes during the course of the day. In addition, constrains (40) introduce effective cuts to the corresponding MILP problem, reducing the total execution time.

As already discussed in the Introduction, the main drawback of the proposed unified UC-ED model with 24-h look-ahead capability is its computational requirements. The test results have demonstrated that the model can solve a medium sized real system, comprising several tens of resources and several hundreds of nodes using DC network representation including contingencies, within 1 to 2 min on a high-end PC. However, at present our model cannot solve within the available tight timeframe US RTO/ISO sized systems comprising several hundreds of resources, few thousands of nodes using AC network representation including contingencies. Advances in optimization software and computer hardware are required for our model to be applied to large sized power systems. However, if the same concept of the unified UC-ED is used with a shorter scheduling horizon of the order of 4-6h (unified real-time ED and real-time UC) our model could solve larger problems within the available timeframe.

\section{CONCLUSIONS}

A MILP-based multiple time resolution unit commitment model has been developed for the solution of the unified unit commitment and economic dispatch problem in systems with high renewable penetration. The unified UC-ED is executed on a rolling basis every $5 \mathrm{~min}$ and provides financially binding dispatch and ex-ante locational marginal prices (LMPs) for the next 5-min interval and advisory UC, dispatch schedule and prices for the remaining scheduling horizon. The proposed model was tested on the medium sized Greek power system and proved to provide adequate capacity and ramping capability to follow abrupt variable generation changes within reasonable execution times in contrast to the conventional UC/ED method which was unable to follow abrupt VG variations. Research is underway for the incorporation of VG uncertainties in the unified UC-ED model.

\section{ACKNOWLEDGMENT}

The authors would like to thank Dr. A. Papalexopoulos, CEO and founder of ECCO International, Inc., for important advice on the latest developments of the U.S. electricity markets. They also thank M. Aimoniotis, I. Blanas, E. Kalfaoglou, I. Tolias, and K. Stavropoulou of the Hellenic Independent Power Transmission Operator for providing the Greek power system data.

\section{REFERENCES}

[1] A. J. Wood and B. F. Wollenberg, Power Generation, Operation and Control. New York, NY, USA: Wiley, 1996.

[2] S. Stoft, Power Systems Economics: Designing Markets for Electricity. Piscataway, NJ, USA: IEEE Press/Wiley, 2002.

[3] L. Xie, P. M. S. Carvalho, and L. A. F. M. Ferreira et al., "Wind integration in power systems: Operational challenges and possible solutions," Proc. IEEE, vol. 99, no. 1, pp. 214-232, Jan. 2011.

[4] B. C. Ummels, M. Gibescu, and E. Pelgrum et al., "Impacts of wind power on thermal generation unit commitment and dispatch," IEEE Trans. Energy Convers., vol. 22, no. 1, pp. 44-51, Mar. 2007.

[5] F. Bouffard and F. D. Galiana, "Stochastic security for operations planning with significant wind power generation," IEEE Trans. Power Syst., vol. 23, no. 2, pp. 306-316, May 2008

[6] A. Papavasiliou, S. S. Oren, and R. P. O’Neill, "Reserve requirements for wind power integration: A scenario-based stochastic programming framework," IEEE Trans. Power Syst., vol. 26, no. 4, pp. 2197-2206, Nov. 2011.

[7] P. A. Ruiz, C. R. Philbrick, E. Zak, K. W. Cheung, and P. W. Sauer "Uncertainty management in the unit commitment problem," IEEE Trans. Power Syst., vol. 24, no. 2, pp. 642-651, May 2009.

[8] A. Tuohy, P. Meibom, and E. Denny et al., "Unit commitment for systems with significant wind penetration," IEEE Trans. Power Syst., vol. 24, no. 2, pp. 592-601, May 2009.

[9] D. Menniti, N. Scordino, and N. Sorrentino, "Secure and economic management of a power system in the presence of wind generation," Elect. Power Syst. Res., vol. 80, no. 11, pp. 1375-1383, Nov. 2010.

[10] J. Wang, A. Botterud, and R. Bessa et al., "Wind power forecasting uncertainty and unit commitment," Appl. Energy, vol. 88, no. 11, pp. 4014-4023, Nov. 2011.

[11] E. M. Constantinescu, V. M. Zavala, and M. Rocklin et al., "A computational framework for uncertainty quantification and stochastic optimization in unit commitment with wind power generation," IEEE Trans. Power Syst., vol. 26, no. 1, pp. 431-441, Feb. 2011.

[12] J. Wang, M. Shahidehpour, and Z. Li, "Security-constrained unit commitment with volatile wind power generation," IEEE Trans. Power Syst., vol. 23, no. 3, pp. 1319-1327, Aug. 2008.

[13] R. Jiang, J. Wang, and Y. Guan, "Robust unit commitment with wind power and pumped storage hydro," IEEE Trans. Power Syst., vol. 24, no. 2, pp. 800-810, May 2012.

[14] L. Wu and M. Shahidehpour, "Comparison of scenario-based and interval optimization approaches to stochastic SCUC," IEEE Trans. Power Syst., vol. 27, no. 2, pp. 913-921, May 2012.

[15] Q. Wang, Y. Guan, and J. Wang, "A chance-constrained two-stage stochastic program for unit commitment with uncertain wind power output," IEEE Trans. Power Syst., vol. 27, no. 1, pp. 206-215, Feb. 2012.

[16] P. Meibom, R. Barth, and B. Hasche et al., "Stochastic optimization model to study the operational impacts of high wind penetrations in Ireland," IEEE Trans. Power Syst., vol. 26, no. 3, pp. 1367-1379, Aug. 2011.

[17] H. Hui, C. N. Yu, R. Surendran, F. Gao, and S. Moorty, "Wind generation scheduling and coordination in ERCOT Nodal market," in Proc. IEEE Power and Energy Soc. General Meeting, San Diego, CA, USA, Jul. 22-26, 2012.

[18] K. W. Cheung and R. R. Rios-Zalapa, "Smart dispatch for large grid operations with integrated renewable resources," in Proc. 2011 Innovative Smart Grid Technology (ISGT) Conf., Jan. 2011. 
[19] Look Ahead Commitment Stage 1-Highlights From Functional Design Document v1.1, Midwest ISO, May 4, 2010. [Online]. Available: https://www.midwestiso.org/WHATWEDO/STRATEGICINITIATIVES/Pages/LookAhead.aspx.

[20] K. H. Abdul-Rahman, H. Alarian, and M. Rothleder, "Enhanced system reliability using flexible ramp constraint in CAISO market," in Proc. IEEE Power and Energy Soc. General Meeting, San Diego, CA, USA, Jul. 22-26, 2012.

[21] Integration of Variable Energy Resources, Order No. 764, Final Rule, Docket No. RM10-11-000, Federal Energy Regulatory Commission, Jun. 22, 2012. [Online]. Available: http://www.ferc.gov/whats-new/ comm-meet/2012/062112/E-3.pdf.

[22] FERC Order 764 Compliance. 15-Minute Scheduling and Settlement. Draft Final Proposal, California ISO, Mar. 26, 2013. [Online]. Available: http://www.caiso.com/Documents/DraftFinalProposal-FERC-Order764MarketChanges.pdf.

[23] V. M. Zavala, A. Botterud, E. Constantinescu, and J. Wang, "Computational and economic limitations of dispatch operations in the next-generation power grid," in Proc. IEEE Conf. Innovative Technologies for an Efficient and Reliable Electricity Supply (CITRES), Sep. 2010.

[24] C. K. Simoglou, P. N. Biskas, and A. G. Bakirtzis, "Optimal self-scheduling of a thermal producer in short-term electricity markets by MILP," IEEE Trans. Power Syst., vol. 25, no. 4, pp. 1965-1977, Nov. 2010.

[25] "MATLAB Version R2012a (7.14.0.739)," The MathWorks Inc., Natick, MA, USA, 2012.

[26] A. Brooke, D. Kendrick, and A. Meeraus, GAMS: A User's Guide. Redwood City, CA, USA: Scientific Press, 1990. [Online]. Available: http://www.gams.com/docs/document.htm.

[27] The IBM ILOG CPLEX Optimizer Website, 2012. [Online]. Available: http://www-01.ibm.com/software/integration/optimization/cplex-optimizer.

[28] J. E. Price and M. Rothleder, "Recognition of extended dispatch horizons in California's energy markets," in Proc. IEEE Power and Energy Soc. General Meeting, Detroit, MI, USA, Jul. 24-28, 2011.

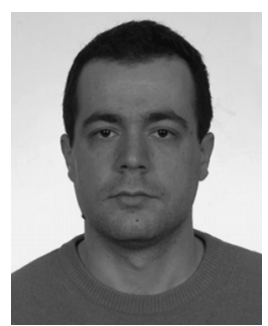

Emmanouil A. Bakirtzis (S'08) received the Dipl.Eng. degree from the Aristotle University of Thessaloniki, Greece, in March 2010. Since February 2012, he has been pursuing the Ph.D. degree in Aristotle University of Thessaloniki.

His research interests are in power system operation, analysis, planning and control.

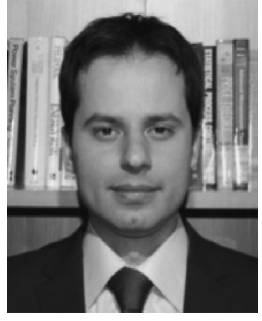

Pandelis N. Biskas (S'01-M'04) received the Dipl.-Eng. and Ph.D. degrees from the Department of Electrical Engineering at the Aristotle University of Thessaloniki, Greece, in 1999 and 2003, respectively.

From March 2005 until July 2009 he was a power system specialist at the Hellenic TSO, Market Operation Department. Currently, he is a Lecturer at the Aristotle University of Thessaloniki, in the Department of Electrical and Computer Engineering. His research interests are in power system operation and control, electricity market operational and regulatory issues, and transmission pricing.

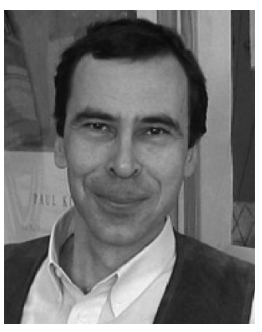

Dimitris P. Labridis (S'88-M'90-SM'00) received the Dipl.-Eng. and Ph.D. degrees from the Department of Electrical Engineering at the Aristotle University of Thessaloniki, Greece, in 1981 and 1989, respectively.

Since 1986 he has been with the Electrical Engineering Department, Aristotle University of Thessaloniki, where he is currently a Professor. His research interests are power system analysis with special emphasis on the simulation of transmission and distribution systems.

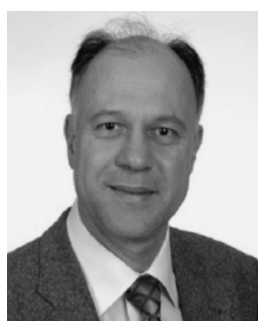

Anastasios G. Bakirtzis (S'77-M'79-SM'95) received the Dipl.-Eng. degree from the National Technical University, Athens, Greece, in 1979 and the M.S.E.E. and Ph.D. degrees from Georgia Institute of Technology, Atlanta, GA, USA, in 1981 and 1984 , respectively.

Since 1986 he has been with the Electrical Engineering Department, Aristotle University of Thessaloniki, Greece, where he is currently a Professor. His research interests are in power system operation, planning and economics. 